\title{
A novel non-invasive, non-conductive method for measuring respiration
}

\author{
Jan Ringkamp ${ }^{1}$, Philipp Radler ${ }^{1}$, Philipp Lebhardt', Jens Langejürgen ${ }^{1}$ \\ ${ }^{1}$ Fraunhofer IPA, Theodor-Kutzer-Ufer 1-3 (Cubex41), 68167 Mannheim, Germany
}

\begin{abstract}
We present a novel method for measuring dynamic changes in thoracic parameters due to breathing based on the coupling of two UHF antennas. The phase shift between the antennas as well as the resonance frequency of the coupled antenna pair change with the air volume in a lung phantom. For evaluation, we built a dynamic 3D printed phantom encasing two compartments separated by an elastic diaphragm. By filling this artificial lung with air the effective permittivity in the compartment changes resulting in a detectable phase and frequency shift. We use a vector network analyzer to show that there is a strong linear dependency between the volume of air in the artificial lung and the frequency at which the $S_{21}$ parameter peaks $\left(R^{2}=0.98\right)$. Related to this, we observe a phase shift $(\Delta \varphi)$ when looking at a single frequency close to the resonance frequency of the system. This shift is also linearly dependent on the volume of air in the lung $\left(R^{2}=0.998\right)$. The presented method is a promising candidate to overcome some of the most important technical burdens of measuring respiratory parameters and might be used as trigger for patient-ventilator synchronization in infants and neonates.
\end{abstract}

Keywords: plethysmography; respiration, antenna detuning, noncontact monitoring, electromagnetic measurement

\section{Introduction}

Respiratory parameters like breathing frequency, tidal volume or inhalation onset are among the most crucial parameters in intensive care. Medical respirators need current values to adjust timing and flow rates in order to ensure an optimal and lung protecting ventilation. This is especially important when it comes to the ventilation of infants or neonates. Here, low tidal volumes (as low as $3 \mathrm{ml}$ ), the comparably higher breathing frequency, and the sensitive skin hamper precise measurement and control of respiratory parameters. When ventilating premature infants, it is common practice to use uncuffed endotracheal tubes to prevent the development of ventilator induced lung injury, like volu- or barotrauma [1]. This poses an additional challenge for respiration sensors, because the unknown leakage due to the uncuffed endotrachael tube makes simple pressure and airflow measurement highly unreliable.

Electrical and electromagnetical measurements are a promising class of sensors, because they are leakage independent and several embodiments can measure without direct contact preventing damage to the infant's sensitive skin.

Uesawa et al. use an electromyogram in [2] to detect muscle activity necessary for diaphragm movement. In this work, an infant was placed on a mattress with integrated sheet-type electrodes. The electrodes function as capacitor plates deriving an ECG and an EMG. The sensing electrodes are connected to a boosttrap buffer to provide a high-impedance input leading to high sensitive. The EMG signal of Uesawa et al. was closely correlated $(r=0.96)$ to the readings of a commercially available belt transducer.

A different approach used in several works is using the patient as the dielectric medium of a plate capacitor:

Teichmann et al. present a sensor in [3], where they used the detuning of a Colpitts oscillator to measure the respiration. Two methods were described. The first system uses a coil as sensing element. Therefore, the patient's respiration changes the inductances of the oscillator by inductive coupling. The second system uses two capacitor plates as sensing elements. In this case, the patient's respiration changes the permittivity between the plates, therefore changing the capacitance and detuning the oscillator. A similar system is described by Kundu et al. in [4]. Kundu et al. use two capacitor plates as well, whereas the plates are made of a conductive textile and integrated in a T-shirt. Both system come with the disadvantage of omnidirectional sensing, especially in the approach of Teichmann et al. 
Because the capacitor plates are mounted in the same plane in [3], there is no distinction between an object in front or behind the plates. Kundu et al. partly overcome this issue by placing the patient between the capacitor plates. Still, in the approach of Kundu et al. a body with high permittivity, e.g. an arm, forms a parasitic capacitance to ground and disturbs the respiration measurement.

In this paper, we present a new kind of sensor system in the class of electromagnetic respiration sensors by using two coupled antennas as sensor element. The issue of omnidirectional sensing can be resolved by coupling the antennas and by carefully choosing their radiation pattern. However, in this paper we only investigate the feasibility of a coupled antenna pair as sensing element under ideals conditions. The radiation pattern of the antennas is not investigated to form a welldefined region of interest, yet.

\section{Theory}

The theory of operation of our sensor system is based on the change of the propagation speed of an electromagnetic signal when passing through a medium [5]. The propagation speed in the medium mainly depends on the mediums permeability and permittivity. The propagation speed in a medium can be calculated as follows [5]:

$$
v=\mathrm{c}_{0} / \sqrt{\mu_{r} \varepsilon_{r}}
$$

where $c_{0}$ is the speed of light in a vacuum, $\varepsilon_{r}$ and $\mu_{\mathrm{r}}$ are the relative permittivity and the relative permeability of the medium.

Because the wavelength $\lambda$ and the frequency $f$ of the electromagnetic signal are hardly linked with the propagation speed in the medium, one can derive from Eq. (1) the wavelength in the medium[5]:

$\lambda=\mathrm{v} / \mathrm{f}=c_{0} /\left(f \sqrt{\mu_{r} \varepsilon_{r}}\right)$

This well-known relation is used, e.g. to reduce the mechanical length of antennas [6] at fixed frequencies.

We use this change in wavelength when passing through a dielectric medium to measure changes in dielectric properties. To do so, we detect the phase shift of an electromagnetic wave transmitted by a first antenna when received by a second antenna. By increasing the permittivity of the medium between the two antennas, the wavelength at a constant frequency decreases and the phase difference between transmitted and received signal increases.

A second effect we have to consider when using antennas that are in close connection to a dielectric material is the change in resonance frequency with the dielectric properties. By solving Eq. (2) for the frequency f, one gets:

$f=c_{0} /\left(\lambda \sqrt{\mu_{r} \varepsilon_{r}}\right)$

The effect arising from Eq. (3) is well-known and can be rather undesired because in common practice it leads to antenna detuning. This is especially the case for body-worn antennas in wearables $[7,8]$, because the body has a rather high permittivity.

In this paper, we investigate the effects described by Eq. (2) and Eq. (3) as two independent means to measure respiration. When considering the human respiration the permittivity of the thorax strongly changes while breathing, because the lung with its rather high permittivity is flooded with air, which has a low permittivity. In the case of Eq. (2), the frequency is fixed and all variables except for the relative permittivity can be considered constant in the application as respiration sensor. The speed of light $c_{0}$ is a constant, the frequency is fixed by the user and the permeability of air and most biological material is the same [9]. In case of Eq. (3), the antennas dimension fixes the wavelength $\lambda$ and we vary the frequency to find the resonance frequency of the coupled antenna pair. So Eq. (3) can be simplified to:

$f=\mathrm{k} / \sqrt{\varepsilon_{r}}$

Where $k=c_{0} /\left(\lambda \sqrt{\mu_{r}}\right)$.

As mentioned earlier, we used a coupled antenna pair as sensor element. Coupled in this case means that the impedance of the first antenna is not only dependent on its selfimpedance but also on the impedance of the second antenna, both antennas are linked by a mutual impedance $\mathrm{Z}_{12}[10]$. The coupling of two antennas depends on their distance, where two antennas far from each other can be consider loosely coupled [10]. The mutual impedance $\mathrm{Z}_{12}$ of two small electric dipoles of the same length can be modeled in the following way [11]:

$Z_{12}=\frac{l^{2}}{4 \pi r^{2}}[\underbrace{\frac{1}{j \omega \cdot \varepsilon \cdot r}}_{C}+R_{0}+\underbrace{j \omega \cdot \mu \cdot r}_{L}] e^{-j 2 \pi r / \lambda}$

Where $\mathrm{l}$ is the dipole length, $\mathrm{r}$ the distance between the dipoles and $\mathrm{R}_{0}$ the wave resistance in free space.

From Eq. (5) an equivalent circuit consisting of a capacitor, a resistor and an inductor can be derived to model the mutual impedance $\mathrm{Z}_{12}$ [11]. Where the capacitor dominates in the antennas near-field and the inductor in the antennas farfield [11]. Because the focus of our sensor systems lies in the detection of permittivity change, it is obvious from the equivalent circuit of the mutual impedance to bring the antennas 


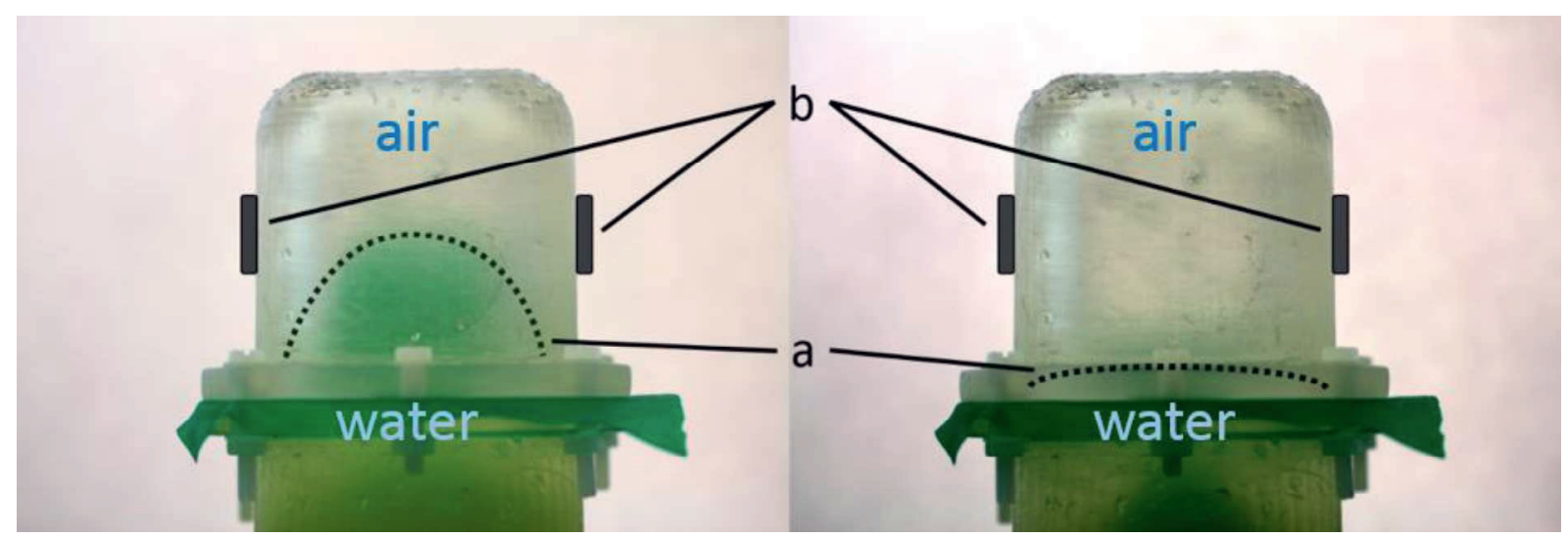

Fig. 1: The lung phantom consists of two compartments. The lower compartment is filled with water and contains a balloon. By inflating the balloon, the water is pushed in the second compartment and an elastic diaphragm (a, dotted line) between the two compartments dilates. This case is shown in the left picture. This models an exhaled state of the lung. By deflating the balloon, the diaphragm contracts. This models an inhaled state and is shown in the right picture. The gray blocks (b) indicate the position at which the antennas were placed.

close together and operate them in or close to their respective near-field.

\section{Material and Methods}

As sensor elements, we used two identical UHF antennas (Molex 105262-0002). The antennas have a transmission band starting at $863 \mathrm{MHz}$, specified by the manufacturer. The antenna is a slightly meandered dipole with a physical length of $79 \mathrm{~mm}$ and a width of $10 \mathrm{~mm}$. We measured scattering parameters, most importantly the $S_{21}$ parameter describing the transmission between the two antennas using a vector network analyzer. The measured frequency range was $10 \mathrm{MHz}$ to $1200 \mathrm{MHz}$ with a step size of $100 \mathrm{kHz}$.

As model for an infant's lung, we fabricated a plastic phantom by $3 \mathrm{D}$ printing. We printed two compartments using a Form 2 3D-printer with clear resin from Formlabs Inc. The outer dimensions of the phantom are $135 \times 76 \times 65 \mathrm{~mm}$ with a wall thickness of $2.4 \mathrm{~mm}$.

An elastic diaphragm separates the two compartments. The lower compartment contains water to simulate the electrical properties of an infant's thoracic tissue and a single balloon. The inflated balloon pushes the water into the upper compartment by dilating the diaphragm. The upper compartment contains air and in conjunction with the diaphragm serves as the model for the infant's lung, where a dilated diaphragm models an exhaled state of the lung and a diminished diaphragm an inhaled state, see Fig. 1.

The balloon was inflated using a $20 \mathrm{~mL}$ syringe held by two clamps mounted on a rod. The second clamp holding the syringe's piston was movable and was used to inflate the balloon with up to $20 \mathrm{~mL}$ with a step size of $1 \mathrm{~mL}$.

\section{Results}

Before we investigated the effect of the lung volume on the antenna pair, we conducted a series of experiments to characterize the antennas. Here, the emphasis was to evaluate the effect of the coupling between the two antennas in free space. The results are shown in Fig. 2. We characterized each antenna using its $S_{11}$ parameter. In a first setup, we measured $S_{11}$ of each antenna without the other antenna in its vicinity. In this case, the antennas are loosely coupled or uncoupled. The two resonances of the each transmission band were clearly visible in the $S_{11}$ parameter. The two resonances were slightly higher than the center frequencies stated by the manufacturer, probably due to different mounting conditions in our setup. In a second setup both antennas were placed in close vicinity to each other. In this case the antennas are coupled. Due to the coupling the resonances in $S_{11}$ of the first antenna shifted to slightly lower frequencies, but more importantly a third resonance was visible around $290 \mathrm{MHz}$ at a much lower frequency than the other two resonances. This resonance arising from the coupling is especially prominent when looking at the $S_{21}$ parameter of the antenna pair. We focused on the lower frequency resonance, because it arose from coupling the two antennas and is related to the mutual impedance. In addition, as shown in equation (5), the mutual impedance has a retardation term depending on the ratio of antenna distance to wavelength. Therefore, a higher wavelength (i.e. lower frequency) leads 
to a more dominant mutual impedance and a higher sensitivity on permittivity changes.

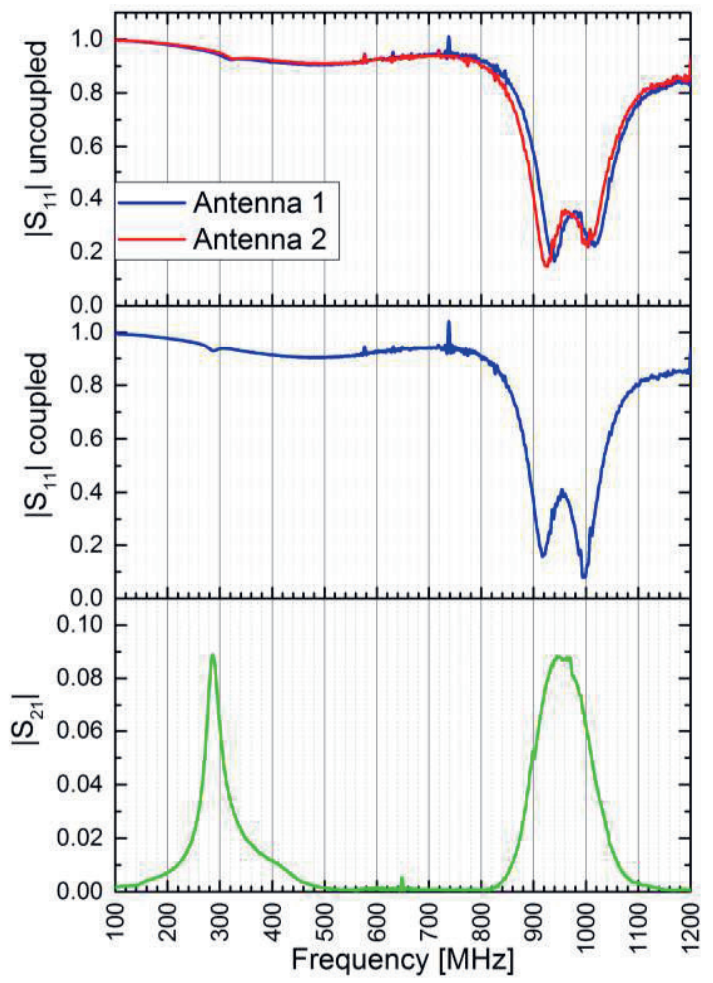

Fig. 2: Coupling of two antennas in free space. The upper diagram shows the $S_{11}$ parameter of the two antennas without the other antenna in its vicinity, respectively. In this case both antennas are uncoupled. Clearly visible are the two resonance frequencies for the antennas two transmission bands between 900 $\mathrm{MHz}$ and $1100 \mathrm{MHz}$. The middle diagram shows the $S_{11}$ parameter of the first antenna, when the second antenna is in its close vicinity. In this case, both antennas are closely coupled. It can be seen that the resonance frequencies above 900 $\mathrm{MHz}$ shift to slightly lower frequencies compared to the uncoupled case. Also, a very small resonance around $290 \mathrm{MHz}$ is visible in the coupled case. The lower diagram shows the $S_{21}$ parameter of the coupled antenna pair. The small resonance in $S_{11}$ around $290 \mathrm{MHz}$ is far more prominent when looking at the $S_{21}$ parameter.

The low frequency resonance shifted to even lower frequencies around $240 \mathrm{MHz}$ to $250 \mathrm{MHz}$, when the antenna pair was mounted on the phantom. The high permittivity of the phantom's water-filled compartment detuned the antenna pairs mutual resonance, as predicted by Eq. (4).

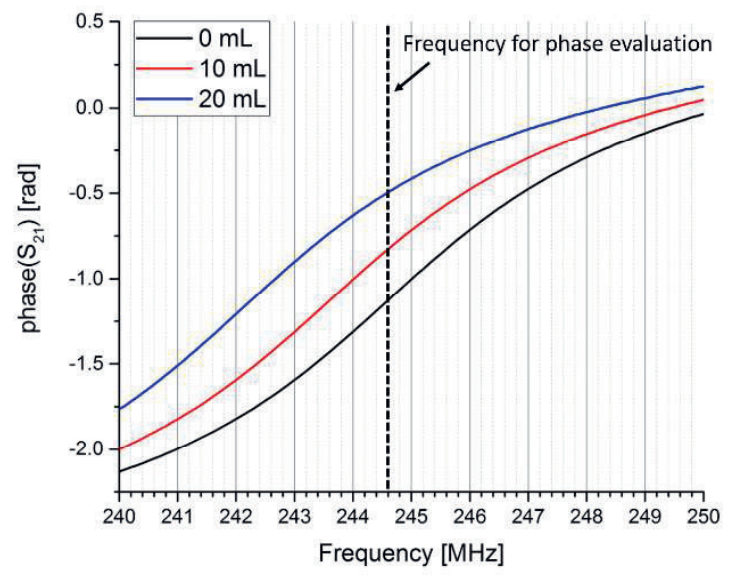

Fig. 3: Phase response for three different water volumes pushed in the upper compartment. The increase of permittivity by pushing water in the upper compartment significantly increases the phase when the evaluation frequency (dotted line) is fixed.

Fig. 3 depicts the phase response for varying water volumes in the upper compartment in the frequency range around the lower resonance frequency. For the evaluation of the phase, we used the resonance frequency when no water was pushed in the upper compartment, as indicated by the dotted line in Fig. 3. It is clearly visible that the permittivity increase leads to a phase increase as predicted by Eq. (2).

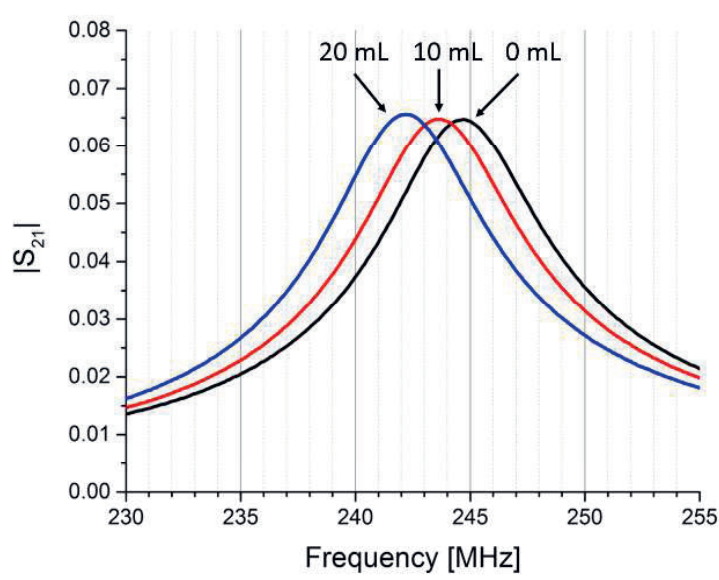

Fig. 4: Resonance in the lower frequency range for three different water volumes pushed in the upper compartments. Clearly visible is the shift of the resonance frequency to lower frequency, when the water volume is increased.

Fig. 4 depicts the resonance in the lower frequency range for varying water volumes in 
the upper compartments. It is clearly visible that the resonance frequency shifts to lower frequencies by increasing the water volume. This matches the prediction made by Eq. (4). The phase at a fixed frequency and the shifted resonant frequency versus the volume of water pushed in the upper compartments are shown in Fig. 5 and Fig. 6, respectively.

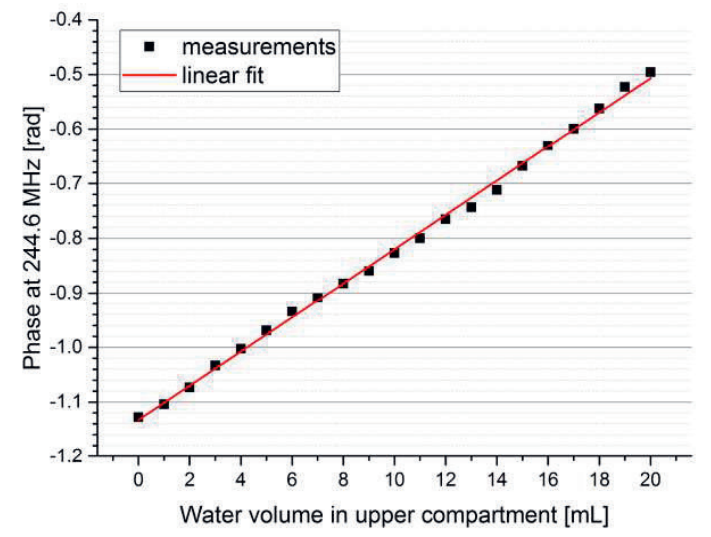

Fig. 5: $\quad$ Phase at constant frequency vs. water volume pushed in the upper compartment. The data was fitted with a linear fit (red curve) yielding $R^{2}=0.9978$.

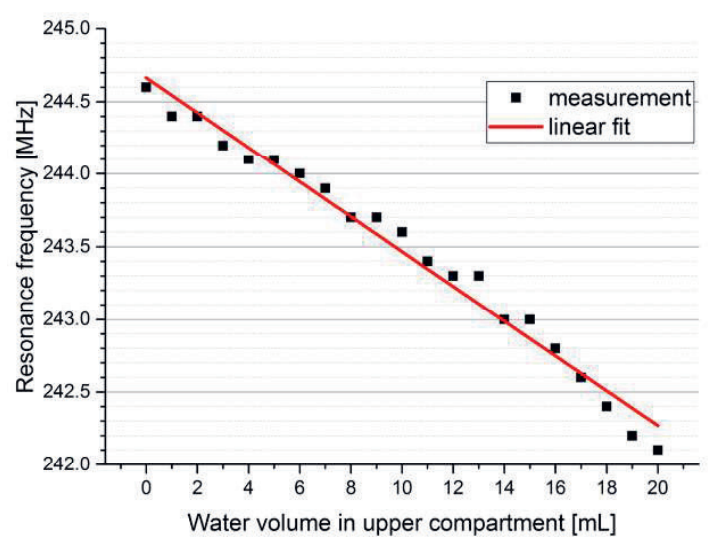

Fig. 6: Resonance frequency vs. water volume pushed in the upper compartment. The data was fitted with a linear fit (red curve) yielding $R^{2}=0.98$

\section{Discussion}

We fitted the data shown in Fig. 5 and Fig. 6 linearly to evaluate our results. The linear fits are shown in the figures as red lines. The linear fit of the phase at constant frequency yielded a coefficient of determination of 0.9978 . The root-mean-square error is $0.00887 \mathrm{rad}$, the slope of the linear fit is 0.0312 rad per $\mathrm{mL}$. The root-mean-square error and the slope therefore yield a resolution of $0.28 \mathrm{~mL}$.
The linear fit of the resonance frequency versus the water volume yielded a coefficient of determination of 0.98 . The root-mean-square error of the resonance frequency fit is $0.104 \mathrm{MHz}$, the slope of the fit is $-0.12 \mathrm{MHz}$ per $\mathrm{mL}$. The root-mean-square error and slope of the resonance frequency fit therefore yield a resolution of $0.87 \mathrm{~mL}$.

The phase at a constant frequency shows a significantly better resolution compared to the resonance frequency, as can be seen in Fig. 5 and Fig. 6. Using the phase instead of the resonance frequency greatly simplifies a later implementation of our sensor system. The resonance frequency as measurement signal would need a smaller step size of the frequency sweep or a fit algorithm to pinpoint the center frequency. Whereas a higher integration time, i.e. more periods, can simply improve the resolution when using the phase as measurement signal. When using the phase only an initial frequency sweep would be necessary to find a suited measuring frequency.

\section{Conclusion}

We presented a new kind of electromagnetic respiration sensor using coupled antennas as sensor element. The sensor was characterized using a 3D printed lung phantom and showed a high sensitivity and linear behavior in the volume range of an infant's lung.

When the sensor is used to assess the absolute volume of a lung, a proper calibration routine probably will be necessary, but this has to be addressed in clinical or pre-clinical trials. However, the proposed sensor might be a viable choice as a trigger for patient-ventilator synchronization in infants and neonates as it can measure dynamic changes in volume with a high temporal and volume resolution. The sensor shows a high sensitivity even at low volume changes and might be well suited to derive breathing patterns in real-time, while being insensitive to leakage. This could strongly reduce the ventilation-induced lung injuries like baro- or volutrauma in the smallest of patients. Future work will include improvement of the antennas as well as non-invasive measurements on adult humans and small rodents.

\section{References}

[1] R. Mahmoud, H. Proquitté, S. Hadhood, G. Schmalisch, Effect of endotracheal tube leakage on respiratory function monitoring: Comparison of three neonatal ventilators, Journal of Pediatric Intensive Care 01, 61-69 (2015); doi: 10.3233/PIC-2012-012.

[2] H. Uesawa, T. Takehara, A. Ueno, Non-contact measurements of diaphragm electromyogram, electrocardiogram and respiratory variations 
with sheet-type fabric electrodes for neonatal monitoring. In: 2018 IEEE EMBS International Conference on Biomedical \& Health Informatics (BHI), 25-28. IEEE; 04.03.2018 - 07.03.2018.

[3] D. Teichmann, J. Foussier, J. Jia, S. Leonhardt, M. Walter, Noncontact monitoring of cardiorespiratory activity by electromagnetic coupling, IEEE Transactions on Biomedical Engineering 60, 2142-2152 (2013); doi: 10.1109/TBME.2013.2248732.

[4] S. K. Kundu, S. Kumagai, M. Sasaki, A Wearable Capacitive Sensor for Monitoring Human Respiratory Rate, Japanese Journal of Applied Physics 52, 04CL05 (2013); doi: 10.7567/JJAP.52.04CL05.

[5] C.-C. Timmermann, Einführung in Leitungen, Vierpole, Transistormodelle und Simulation mit numerischen und symbolischen CAD/CAESystemen: Mit 23 Tabellen. PROFUND-Verl., Plankstadt; 1997.

[6] K. Mizuno, Y. Daiku, S. Ono, Design of Printed Resonant Antennas for Monolithic-Diode Detectors, IEEE Transactions on Microwave Theory and Techniques 25, 470-472 (1977); doi: 10.1109/TMTT.1977.1129136.

[7] J. Buckley, K. G. McCarthy, B. O'Flynn, C. O'Mathuna, The detuning effects of a wrist-worn antenna and design of a custom antenna measurement system. In: 2010 European Wireless Technology Conference (EuWiT 2010): Paris, France, 27 - 28 September 2010; [part of the 13th European Microwave Week 2010 (EuMW 2010), 261-264. Piscataway, NJ: IEEE; 2010.

[8] P. J. Soh, H. A. Sulaiman, M. A. Othman, Abd. Aziz, Mohamad Zoinol Abidin, M. F. Abd Malek, K. Kamardin, M. K. A. Rahim, N. A. Samsuri, M. E. Jalil, N. A. Ahmad, Planar Textile Antennas Performance Under Wearable and Body Centric Measurements. In: P. J. Soh, H. A. Sulaiman, M. A. Othman, Abd. Aziz, Mohamad Zoinol Abidin, M. F. Abd Malek (eds.). Theory and Applications of Applied Electromagnetics, 8195. Cham: Springer International Publishing; 2016.

[9] R. Pethig, D. B. Kell, The passive electrical properties of biological systems: their significance in physiology, biophysics and biotechnology, Physics in Medicine and Biology 32, 933-970 (1987); doi: 10.1088/00319155/32/8/001.

[10] R. King, Coupled Antennas and Transmission Lines, Proceedings of the IRE 31, 626-640 (1943); doi: 10.1109/JRPROC.1943.233357.

[11] H. Wheeler, The Radiansphere around a Small Antenna, Proceedings of the IRE 47, 13251331 (1959); doi:

10.1109/JRPROC.1959.287198. 